\title{
Kernos
}

Revue internationale et pluridisciplinaire de religion grecque antique

19 | 2006

Varia

\section{Fécondité des pratiques catalogiques}

\section{Lambros Couloubaritsis}

\section{OpenEdition \\ Journals}

Édition électronique

URL : https://journals.openedition.org/kernos/458

DOI : $10.4000 /$ kernos.458

ISSN : 2034-7871

Éditeur

Centre international d'étude de la religion grecque antique

Édition imprimée

Date de publication : 1 janvier 2006

Pagination : 249-266

ISSN : 0776-3824

Référence électronique

Lambros Couloubaritsis, "Fécondité des pratiques catalogiques », Kernos [En ligne], 19 | 2006, mis en ligne le 22 mars 2011, consulté le 24 août 2022. URL : http://journals.openedition.org/kernos/458 ; DOI : https://doi.org/10.4000/kernos.458 


\title{
Fécondité des pratiques catalogiques
}

\begin{abstract}
Résumé : Mise en forme des différents procédés énumératifs en partant des listes, en insistant plus spécialement sur la différence entre katalegein (dire les choses successivement) et mytheuesthai (façon de parler autorisée qui crée un effet). L'analyse des formes catalogiques révèle deux pratiques dominantes : les généalogies et les cheminements, tandis que l'histoire du mythos s'achève par une multiplicité d'usages.
\end{abstract}

Abstract : Fecundity of Catalogical Practices. Formation of different enumerative procedures departing from the lists, insisting especially on the difference between katalegein (saying things successively) and myth (an authorised way of speaking, which creates an effect). The analysis of catalogical forms reveals two principal practices: genealogy and routes, while the history of myths ends by a multiplicity of uses.

\section{De l'image au mythe}

Parmi les procédés énumératifs, l'usage des listes est très ancien. La plupart des cultures façonnent aussi bien des listes d'éléments graphiques que des images en série et autres objets servant, entre autres, aux rituels, avec les formulations qui s'y rapportent (dédicaces, dons, épiphanies, etc.). Dans la civilisation grecque la pratique des listes avait intégré l'espace collectif de la cité (agoras, sanctuaires, nécropoles...) ${ }^{1}$. Elle est devenue un élément des réformes de Solon, pour signifier les lois qu'il promulgua ${ }^{2}$. La topologie forme un axe important pour interpréter les procédés séquentiels, qu'ils soient oraux ou écrits. Dans ce genre de production, le contexte socio-politico-religieux est implicite mais peut être dévoilé par une analyse circonstanciée. L'histoire de procédés séquentiels marqués par l'écriture, l'image et la parole n'est pas simple, si l'on songe à la difficulté d'établir l'histoire des transfigurations du logos et du mythos.

Pour simplifier, on peut dire qu'au plus près de la matérialité, dans l'ordre de l'écriture et du graphisme, la pratique des listes concerne tant les tablettes (par exemple mycéniennes) que les images et les objets. Elle s'achève dans les katagraphai de la société bureaucratisée de l'époque hellénistique ${ }^{4}$. À

\footnotetext{
${ }^{1}$ Voir ci-dessus, la présentation du colloque.

${ }^{2}$ Cf. mon étude « Les enjeux du logos : de l'oral à l'écriture », Civilisations 46 (1998), p. 193239.

3 Qui constitue l'un des thèmes de mes recherches sur la pensée grecque et l'histoire de la philosophie depuis trente ans, auquel j'ai consacré une grande partie de mes publications.

${ }^{4}$ L'expression katagraphè désigne des catalogues écrits d'énumération de noms, de biens, de soldats, etc., comme l'indique Plutarque, Vie de Cicéron, 46; Moralia, 482b-c. Voir déjà Platon, Lois, 741c, etc. Cf. l.c. (n. 2). p. 237-239.
} 
Rome, sans se confondre avec la série d'images, les systèmes énumératifs (inventaires et répertoires) servaient à répertorier le patrimoine de la cité, renforçant les procédés sériels d'ordre matériel. Les mises en forme séquentielles sont tributaires de la finalité de chaque procédé et de la façon d'organiser ces listes. Le cas des images fait voir clairement cette perspective, qui excède les procédures techniques et les matériaux utilisés. C'est bien dans cette différence, déjà relevée par Aristote à propos des fonctionnalités du langage (lexis) en la comparant à l'art de la peinture, que réside la question du sens.

Dans sa Poétique il fait voir, par analogie, les trois composantes du dessin et de la tragédie : le moyen, la manière et le rapport à la chose. Tandis que, pour la peinture, la couleur concerne le moyen, le dessin, la manière d'organiser les couleurs, et l'image, le rapport à la chose; pour la tragédie, c'est le langage (lexis) qui constitue le moyen, le mythe, la manière d'arranger le langage, et l'action (et le spectacle), le rapport à la chose. Autrement dit, comme l'image, le «mythe » est compris, non comme un récit mais comme une façon d'arranger les choses dont il est question selon une perspective d'imitation (ou représentation) d'actions ${ }^{5}$. Ainsi, par un subtil glissement, la question de l'image conduit à la question du mythe. Or, depuis Homère, le terme mythos a persévéré en multipliant ses usages au fil du temps, souvent en incluant des procédés séquentiels. D'où la confusion possible avec le discours catalogique.

À une époque tardive, comme celle où se placent les romans étudiés par M. Briand ci-dessus, les Histoires vraies de Lucien révèlent à la fois la liberté de produire des fictions qu'il revendique et le rôle interprétatif du lecteur ${ }^{6}$. Il s'agit là du moment culminant d'une longue histoire, dont on trouve des indices dans le monde archaïque, où l'interprétation était davantage encadrée par des règles de décodage qui nous échappent encore, suivies par des pratiques allégoriques $^{7}$. Au-delà du problème qui lie mythos (au sens large) et interprétation (y compris les procédés de décodage), le roman s'impose par sa capacité de produire des affections. Depuis Homère, le terme mythos en tant que façon de parler qui agence d'une certaine façon le langage, produit des affections sélectives, comme le rire (mythos comique) ou la crainte et la pitié (mythos tragique $)^{8}$. Cette propriété permet de séparer les champs sémantiques

\footnotetext{
5 Voir mon étude «L'institution du langage chez Aristote », Études phénoménologiques 17 (1993), p. 51-69.

${ }^{6}$ Voir, en plus de la communication de M. Briand, ci-dessus, son étude « Lucien et Homère dans les Histoires vraies : pratique et théorie de la fiction au temps de la seconde sophistique », Lalies 25 (2002), p. 127-140. Sur la question de l'interprétation, voir aussi M. DetIENNE, L'invention de la mythologie, Paris, Gallimard, 1981, p. 131 sq.

${ }^{7}$ Voir mon étude, « Y-a-t-il une logique archaïque ? », in F. BEETZ, E. GILLET (éds), Logique en perspective (Mélanges offerts à Paul Gochet), Bruxelles, Ousia, 2000, p. 45-79.

8 Aristote, Poétique VI, 1450a 38 - b3. Voir mes études : l.c. (n. 5); «Statut mythique de l'affectivité et dialectique dans le Phèdre », in La voix des phénomènes (Mélanges G. Florival), Bruxelles, 1995, p. 33-65; "Statut cognitif et affectif du mythe à l'origine de la philosophie », Uranie 9 (2000), p. 9-18 (= J. FABRE-SERRIS, Mythe et Littérature). Cf. aussi S. KLIMIS, Le statut du
} 
du mytheuesthai du katalegein - qui se déploie en dehors d'une connotation affective -, sans quoi le logos n'aurait pu fonder la rationalité argumentative et scientifique. D'où la question qui se tient à l'horizon de la (con)fusion progressive du mythos dans la philosophie et la littérature : comment Homère, qui utilise de nombreux termes pour signifier les nuances du discours', a-t-il compris le terme mythos et ses dérivés?

En Od. XVII, 57, une fois que Télémaque a parlé (ephônèsen), Pénélope regagne ses appartements, sans qu'aucune parole ne s'envole de ses lèvres (apteros epleto muthos) - ou plutôt, selon ce que dit le texte, sa façon de parler était sans ailes, c'est-à-dire qu'elle était silencieuse parce qu'aucune «façon de parler » ne s'envolait de ses lèvres. Cet exemple montre la difficulté de comprendre le terme mythos, qui met en jeu différentes façons de parler, sans rapport avec un récit.

D'autres exemples associent mythos au procédé séquentiel (récit) sans les confondre, comme en $O d$. III, 67 sq., où la mise en scène a lieu au terme d'un dîner. S'adressant à Télémaque, Nestor « commença par ces paroles » (mythôn), en disant que, s'il y a un moment favorable pour interroger les étrangers, c'est quand ils ont joui des plaisirs de la table. Le sens est plutôt : « il commença par ces façons de parler en vue de produire un effet à partir d'un point de vue autorisé », en vue de persuader ou charmer les interlocuteurs $^{10}$. Nestor pose aussitôt une suite de questions sur le mode d'un catalogue. Et Télémaque demande de lui dire/décrire/raconter point par point (katalexô) ce que ses yeux ont vu ${ }^{11}$. Homère utilise ici plusieurs fois muthos /

mythe dans la poétique d'Aristote. Les fondements philosophiques de la tragédie, Bruxelles, Ousia, 1997

9 Cf. H. FOURNIER, Les verbes « dire » en grec ancien, Paris, Klincksieck, 1946.

10 Richard MARTIN interprète mythos comme un discours d'autorité, associé au statut du personnage qui le prononce (The Language of Heroes. Speech and Performance in the "Iliad", Ithaca / Londres, Cornell University Press, 1989 [1992], p. 46-48). L'observation me semble pertinente, mais j'ajouterai que ce qui confère l'autorité à ce type de discours, c'est la façon de parler dont le personnage supérieur s'adresse à des interlocuteurs égaux ou inférieurs, dans le but qu'ils ressentent l'effet de la parole ou du discours

${ }^{11}$ Ce procédé par questions en catalogue a été mis en évidence par Sylvie PERCEAU, La parole vive. Communiquer en catalogue dans l'épopée homérique, Louvain / Paris, Peeters, 2002. Sans sous-estimer la valeur de ce travail, sur de nombreux points remarquable, javoue que certaines thèses suscitent l'embarras. Déjà l'idée de tirer de cette procédure interrogative le statut communicationnel du katalegein soulève des difficultés, surtout sans une analyse de toute la sémantique du langage chez Homère qui, je l'ai dit, met en œuvre un nombre considérable de termes différents. Cette pratique philologique réductionniste se rencontre aussi dans la traduction « dire en catalogue », qui forme une redondance. L'idée traditionnelle de « dire point par point » ou « raconter / décrire successivement » suffisait, comme le montre M. FINKELBERG (« Homer's view of the epic narrative. Some formulaic evidence », CPh 82 [1987], p. 135-138). On pourrait néanmoins ajouter: «dire... en rassemblant», comme cela ressort de l'usage de legô chez Homère (voir infra). Du reste, l'idée que la pratique catalogique concerne un processus de communication, au détriment du dialogue (pourtant présent chez Homère), et investi d'une dimension affective est bien le résultat de cette absence d'une étude des termes, parmi lesquels certains concernent l'affectivité, comme le terme mythos. Dès lors, si l'interrogation catalogique qui appelle une réponse catalogique n'est, comme je le crois, qu'un cas particulier parmi les 
mutheô : 1. Télémaque, qui croit que son père est mort, lui demande de lui parler de sa mort : «L'as-tu vue de tes yeux ? Ou bien as-tu entendu la façon dont un autre l'a façonnée (è allou mûthon akousas plazomenou) ? (...) Ne mets ni des égards, ni ta compassion à m'adoucir les choses mais décris / raconte-moi point par point (êu moi katalexon) ce que tes yeux ont vu ». 2. Quand Nestor lui répond en énumérant les morts célèbres de Troie, y compris son propre fils, il précise qu'ils ont tous subi des souffrances, en s'exclamant : «Quel homme, parmi les mortels, peut trouver une façon de parler de toutes ces choses (ekeina panta ge muthèsaito)?"

Si, dans le premier cas, il est question d'une façon de parler façonnée par quelqu'un (histoire vraie, fable ou autre chose) opposée à une description/récit point par point (katalexon), dans le second cas, on cherche un homme capable de trouver une façon de parler qui convient le mieux pour décrire/raconter de telles choses. À la description successive du katalegein, la sémantique du mythos ajoute, par les diverses façons d'agencer les mots, une dimension affective. En effet, au moment où Nestor dit à Télémaque qu'il devrait rester longtemps chez lui pour l'interroger (exereois) sur les souffrances des Achéens à Troie, il ajoute qu'il se lasserait et rentrerait dans son pays, tout en faisant allusion à Ulysse, qu'il qualifie de « plus ingénieux de tous (les Grecs) ». Et il ajoute qu'il est surpris de constater que leurs façons de parler (muthoi), d'Ulysse et de son fils, se ressemblent. Il s'agit bien sûr, non du contenu du discours mais de la forme, de l'intonation de la voix, des formules utilisées et de la façon de les agencer, qui produisent des effets dans l'âme de celui qui le reçoit.

Quant au rapport de cette sémantique avec la vérité, le passage Od. XVII, 6 sq. révèle que, lors de son retour à Ithaque, Télémaque se trouve à proximité d'Ulysse (sans le reconnaître) qui avait trouvé refuge chez Eumée, leur porcher (qui ne le reconnaît pas non plus). Il veut s'en débarrasser pour descendre en ville visiter sa mère et le confie à Eumée, en disant de le conduire là-bas et que d'autres s'en occupent, car, pour sa part, il ne peut se charger de tout le genre humain. «Si notre hôte le prend en mal, tant pis

multiples usages du katalegô, sa fonction doit être cherchée ailleurs : dans le faire voir la complexité du réel au moyen des procédés de simplification dont font partie les pratiques séquentielles, dont la reconstitution par un redressement rétablit la complexité ordonnée du réel. Pareille démarche est bien objective, ne nécessitant pas toujours une communication, si ce n'est celle de s'adresser à quelqu'un, ni dès lors une référence affective directe, car le discours catalogique est foncièrement dépassionnalisé. Du reste, la co-présence du katalegô et du mytheô milite dans ce sens - contrairement à ce qui est dit à la p. 213, parlant de conjonction « inédite et paradoxale ». Comme le terme katalegein suppose le récit (dire les choses successivement), parler d'« un récit (mython) que tu as dit en catalogue avec compétence » multiplie les redondances. Mythos signifie à l'époque « une façon de parler qui produit un effet » et le contexte du passage fait état du charme produit par cette façon de parler. Ce texte fait voir qu'Ulysse est loué par Alkinoos pour sa façon à la fois de parler (mython), qui est à la hauteur des meilleurs aèdes, et pour sa capacité de raconter savamment ses histoires (epistamenôs katalexas). Il l'incite de continuer à raconter exactement et successivement (atrekeôs katalexon) ses travaux et ses prodiges, car il a envie de passer la nuit à l'écouter, à condition qu'il trouve une façon propice de parler de ses maux (ta sa kèdea mythèsasthai). 
pour lui ! J'aime mon franc-parler (entendez: ma façon habituelle de dire des choses vraies : ê gar emoi phil'alèthea muthèsasthai).

Ce texte marque sa différence avec les autres passages du même contexte, où il est question de raconter la vérité, c'est-à-dire de décrire point par point des événements authentiques (alètheièn katalexô). Mais comme mythos signifie d'abord façon de parler, multipliant les possibilités, il peut aussi prendre le sens de façon de raconter, donc aussi de dire des fables, ou à l'inverse, de dire la vérité. C'est cette propriété remarquable du terme mythos en tant que façon de parler, qui explique pourquoi le terme aura tant d'usages plus tard, du moins à partir de Platon. Nous verrons que ce jeu entre vérité et fiction rencontre une autre structure ambiguë, celle qui est fondée sur l'annonce hésiodique d'après laquelle les Muses disent des «mensonges » (pseudea), et que c'est seulement quand elles le veulent qu'elles font état de situations vraies (Théog., 27-28).

Enfin, pour faire voir davantage le lien entre mythos et affectivité, rappelons le passage de l'Iliade (I, 544 sq.) où Héra, vexée, s'adresse durement à Zeus : «Avec quel dieu encore viens-tu de comploter, perfide ? Tu te plais toujours, loin de moi, à décider d'un cœur secret; et jamais encore tu n'as daigné me dire de toi-même un mot concernant ce que tu penses (eipeîn epos hotti noèsès). » À quoi Zeus répond : "Héra, n’espère pas connaitre toutes mes façons de parler (muthous) ». Et il ajoute : "Même toi, mon épouse, tu auras fort à faire pour y parvenir. S'il est convenable de l'entendre (akouo$m e n)$, il n'est pas un dieu, ni un homme qui les connaîtra avant toi... ». Héra réplique : "Terrible Cronide, quelle est cette façon de parler (muthon eeipes) ? » - en révélant qu'elle avait peur qu'il soit séduit par Thétis.

Pour conclure cette pérégrination du côté du mythos, il faut rappeler que l'usage homérique, que je viens d'esquisser, périclite avec le temps, puisque les occurrences du terme chez Hésiode, Pindare ou Hérodote deviennent progressivement négligeables. Avec Platon, la (con)fusion entre mythos et logos est courante, et c'est pourquoi, lorsque Aristote s'applique à fonctionnaliser le langage, il enferme pour ainsi dire la pratique du mythe dans la Poétique. En revanche, le logos, bien enraciné déjà dans la pensée pré-philosophique, prend de plus en plus d'ampleur sous des formes multiples. Son caractère dépassionnalisé permet d'intégrer davantage sa dimension interne qu'est la raison, en ouvrant à la possibilité de rendre raison de quelque chose selon un ordre successif d'arguments.

\section{Action d'énumérer (arithmô) et katalegô}

Legô dans les textes archaïques signifie, d'une part, l'acte de cueillir (trier, choisir parmi...), de recueillir, de ramasser et, d'autre part, l'acte d'énumérer, de dénombrer, de raconter ${ }^{12}$. Il ., VIII, 507, parle de ramasser/assembler (legesthe) beaucoup de bois, et XXIII, 236 sq., de recueillir les os de Patrocle, en les reconnaissant : "ils se laissent aisément reconnaitre, car ils sont au

${ }^{12}$ Cf. FOURNIER, o.c. (n. 9), p. 53 sq. 
milieu du bûcher, tandis que les autres ont brûlé à part ». En XXI, 26 sq., on apprend que, quand les bras d'Achille étaient las de tuer, il rassembla dans le fleuve, vivants, douze jeunes hommes, qui ont payé pour Patrocle ${ }^{13}$. La transition entre l'action d'énumérer et le katalegein est exprimée quand Ulysse demande à Télémaque d'indiquer le nombre des prétendants et d'exposer leur nom (faire leur généalogie et raconter des éléments significatifs de leur vie) : « age moi mnèstèras arithmèsas katalexon » ( $\mathrm{Od}$. XVI, 235). Et il ajoute : « que je sache combien ils sont et ce qu'ils valent, puis je réfléchirai en mon cœur valeureux et je déciderai si, seuls, nous pouvons les attaquer sans aide ou s'il nous faut aller chercher quelque renfort ». Bref, katalegô est plus qu'une énumération, car il rassemble au fil de l'exposé un ensemble de variables qui lui confèrent son sens.

Hérodote est plus clair encore lorsqu'il dit : «cesse de raconter de vaines histoires (legôn logous mataious) sur les Grecs, qui ne méritent pas d'entendre des récits méprisants sur eux...» (VII, 10). Notons que logos prend ici le sens d'énumération «en rassemblant», de récit, d'histoire, alors que ce même Hérodote utilise ailleurs tantôt katalegein pour raconter et tantôt le même type de structure pour parler d'«arguments» (VIII, 65) ${ }^{14}$. En tout cas, le glissement est décisif pour comprendre qu'une suite d'arguments est analogue au discours catalogique. Où s'accomplit la différence entre discours mythique et discours argumentatif ? En fait, Hérodote utilise indifféremment legein et katalegein pour «raconter», ce qui atteste un mode de discours qui confirme la prédominance future du logos au détriment du katalogos, surtout parce qu'il n'utilise que deux ou trois fois le terme mythos, comme une façon de parler, en l'occurrence la «fable ». Du reste, l'abondance de la sémantique du logos suscite des usages que la philosophie ne manquera pas d'exploiter. En IX, 27, il écrit : " nous savons que les Grecs marchant ensemble (synodos) en ce lieu, se sont rassemblés (syllegènai) pour combattre les barbares et non pour raconter des récits/histoires (ou logôn)». Avec Aristote, syllogismos marque l'avènement du raisonnement. Dans l'histoire de la transmutation du logos qui apparaît en filigrane ici en allant du catalogue au syllogisme, la pensée grecque peut se lire à travers des composés du logos, allant du legô et du katalegô archaïques au dia-legomai, sous ses multiples applications (dialogue et dialectique), jusqu'à la découverte du syl-logizomai. Nous verrons que, dans cette histoire, le rapport entre mythos/discours catalogique et syllogisme s'accomplit dans la nécessité, pour le premier, d'un redressement et dans l'élimination de cette exigence, comme le révèle l'expression orthos $\log o s$, discours correct, droit ou dressé $e^{15}$.

${ }^{13}$ Cf. aussi Il. III, 188; Od. IV, 452; mais aussi au sens d'énumérer en $O d$. III, 240; XIV, 197; Il. II, 222; XIII, 275, 292, etc.

${ }^{14}$ Voir la communication de I. Papadopoulou ci-dessus.

${ }^{15}$ J'ai analysé cette problématique dans un texte en grec moderne : «Le problème du mythe » dans une communication à l'Académie d'Athènes, qui faisait le point de mes recherches jusqu'en 2001, et qui a paru dans le Bulletin de l'Académie d'Athènes 76 (B), 2001, p. 316-340. 
Le point de départ de cette histoire peut être situé dans le célèbre catalogue des vaisseaux de l'Iliade, qui fait voir, par un processus de redressement l'ensemble de la géographie de la Grèce en fonction des cités qui ont participé à l'effort de guerre, mais aussi leurs rois et ce qu'on peut retirer de leurs légendes. Mais en réalité ce texte révèle davantage encore, car l'exposé est amorcé par une référence aux Muses et, en même temps, est intégré dans la mise en intrigue de la guerre et des luttes intestines entre les Achéens. Aristote, qui l'avait d'ailleurs discerné, précise que la composition s'accomplit par une suite d'épisodes ${ }^{16}$, où domine le schème de la violence ${ }^{17}$. Il précise qu'Homère ne traite pas l'ensemble de la guerre de Troie, car les proportions du récit ne seraient pas maîtrisables. Il préfère se limiter à une partie de la guerre et la présenter par épisodes. Dans l'Odyssée, les successions dans la narration sont régulées par le schème du chemin, préservant ainsi la primauté du discours catalogique. Dans le cas des romans étudiés par M. Briand cidessus, bien que l'intrigue soit régie par le schème de l'amour, mis jadis au premier plan par Hésiode et Empédocle, puis par le Banquet et le Phèdre de Platon $^{18}$, la co-présence des logoi et des mythoi sans distinction, assure, à l'instar de l'Iliade, la prédominance de «l'axe mythologique », composé d'un ensemble de formes narratives hétéroclites de la culture grecque, au détriment des catalogues ${ }^{19}$.

\footnotetext{
${ }^{16}$ Poét. XXIII, 1459a 30-37.

${ }^{17}$ Par « schème », j'entends une expérience proche et concrète (etymon), comme la parenté, le chemin, l'amour, la violence, l'artisanat..., - prise comme condition de régulation d'une forme narrative ou d'un catalogue. La problématique des schèmes et de leur transmutation en modèles est un des fils conducteurs de mes recherches sur le mythe, que j'ai prolongées jusque dans la pratique philosophique contemporaine, où domine le schème du jeu ( $c f$. «La question du jeu du monde ", Rue Descartes 18 [1997], p. 51-85). Cette approche permet de départager différentes pratiques du mythe et de suivre également leurs transformations historiques ( $c f$. « Le statut du mythe dans l'histoire de la philosophie ", Sartoniana 11 [1998], p. 15-41). Prolongeant le schème du jeu, j'ai proposé moi-même sa complexification grâce au modèle du «thêâtre », sur lequel est organisé le déroulement de mon dernier livre sur La proximité et la question de la souffrance bumaine, Bruxelles, Ousia, 2005.

${ }^{18}$ Sur l'ampleur de l'éros, voir Cl. CALAME, L'Éros dans la Grèce antique, Paris, Belin, 1996.

${ }^{19}$ La formation d'un axe mythologique, à côté d'un axe généalogique et d'un axe topologique a été l'une des premières réponses que je me suis permis d'élaborer pour sortir de l'impasse où nous avaient conduits, non sans raison, P. Smith et M. Detienne. Voir, entre autres, mes études : "De la généalogie à la généséologie », in J.-F. MATtei (éd.), La naissance de la Raison en Grèce, Paris, PUF, 1990, p. 83-96; « Le statut transcendantal du mythe », in G. Florival (éd.), Figures de la rationalité, Paris / Louvain, Vrin / Peeters, 1991, p. 14-44; "Transfigurations du paradigme de la parenté ", in J. GAYON et J.-J. WunENBURGer (éds), Le paradigme de la filiation, Paris, L'Harmattan, 1995, p. 169-186; "Genèse et structure dans le mythe hésiodique des races ", in F. Blaise, P. Judet de la Combe, Ph. Rousseau (éds), Le métier du mythe, Lille, Septentrion, 1996, p. 475-518, ainsi que l'Introduction de mon livre Mythe et philosophie chez Parménide. Bruxelles, Ousia, $1990^{2}$ [1986], p. 45-46. J'ai proposé une première synthèse dans la première partie de mon Histoire de la philosophie ancienne et médiévale, Paris, Grasset. 1998. Depuis, j’ai amélioré ce modèle en soumettant la généalogie à l'axe catalogique et en ajoutant un quatrième axe qui tient compte de divers types de temps, que je qualifie désormais d'axe chronologique. Bien plus, j'ai élaboré un modèle parallèle pour le rite, en intégrant dans l'axe catalogique les épreuves
} 
Cette aptitude des procédés séquentiels à dominer le discours ou au contraire à se plier à la force de l'intrigue, révèle la difficulté de départager les champs du langage dans la culture antique. Pour la clarté de l'analyse, il convient de souligner que : $1^{\circ}$ dès lors qu'il y a succession séquentielle susceptible d'une unification conceptuelle, on peut parler d'axe catalogique; et $2^{\circ}$ selon les schèmes utilisés la forme catalogique se divise en plusieurs espèces. Ainsi, avec le schème de la parenté, on édifie des généalogies, avec celui du chemin, des voyages ou, dans le cas du rite, des opérations successives ou des initiations, et dans le cas du savoir, des méthodes. Avec le schème de l'artisan, on produit des mythes de création, comme celui du démiurge de Platon, qui utilise, en parallèle, deux approches catalogiques : le processus mythique de création du monde et le développement mathématique qui lui correspond (succession de nombres, de figures et de volumes pour le Corps du monde, et de proportions harmoniques pour l'Âme). D'autre part, l'étude de la structure narrative des généalogies montre que le schème de la parenté est souvent associé aux schèmes de la violence et de l'amour/sexe. Or, après une certaine éclipse dans la philosophie classique, le schème de la parenté a retrouvé une nouvelle vigueur sous une forme limitée (père-fils) dans le christianisme, depuis la rencontre entre l'hellénisme et le judaïsme ${ }^{20}$. Il se perpétue aujourd'hui avec l'irruption de la question du sujet, à travers la psychanalyse freudienne ${ }^{21}$, alors qu'il est devenu secondaire dans les formes narratives dominantes, au contraire de la violence narrative qui est encore très présente, avec le sexe, dans les romans et les autres formes narratives, transposés en images à la télévision et au cinéma.

Ces différentes approches peuvent trouver un point de convergence à travers une architectonique complexe qui met en jeu quatre axes complémentaires: le catalogique, le mythologique, le topologique et le chronologique, dont chacun se compose de diverses variantes ${ }^{22}$.

1. L'axe catalogique inclut un ensemble de pratiques séquentielles, y compris les généalogies et les voyages, et va aboutir au discours argumentatif et aux procédés de déduction, qui utilisent respectivement des suites d'arguments ou de conclusions à partir d'étapes intermédiaires, dont le moyen terme dans le syllogisme constitue la première découverte majeure dans l'histoire de la raison ${ }^{23}$

successives, les rites de passage, les initiations, etc., et en réaménageant l'axe mythologique en un axe opérationnel, où l'on trouve cérémonies, sacrifices, prières, transes, sacrements, etc.

${ }^{20}$ Voir mes histoires de la philosophie : Aux origines de la philosophie européenne, Bruxelles, De Boeck, $2003^{4}$ (1992) et Histoire de la philosophie ancienne et médiévale, déjà citée, ainsi que les deux articles cités (1990 et 1995) à la note 19

${ }^{21}$ Voir mon étude "Schèmes de la parenté et du miroir dans la psychanalyse », in F. MARTENs (éd.), Que reste-t-il de nos amours? Revue de l'Université de Bruxelles (2000), p. 53-70.

${ }^{22}$ Pour l'histoire de la constitution de cette architectonique, voir la note 19, ci-dessus.

${ }^{23}$ On oublie souvent que la découverte des syllogismes et l'étude de la validité est l'une des premières grandes découvertes de notre logique et qu'elle a valu à Aristote beaucoup de travail et de peine, comme il le révèle lui-même dans ses Réfutations sophistiques, 34. 
2. L'axe mythologique se compose d'un ensemble de formes narratives (contes, légendes, fables, épopées, etc.). Entre l'épopée (qui utilise diverses formes narratives, y compris l'ainos) et les romans, se déploient des discours remarquables comme les tragédies ou les comédies ${ }^{24}$.

3. L'axe topologique utilise tous les lieux possibles, dans le visible et l'invisible, et dont le transfert sur le plan même du langage a produit une cartographie connue par les « lieux » du langage, avec un point culminant les Topiques et la Rhétorique d'Aristote.

4. Enfin, l'axe chronologique, intègre dans l'ordre temporel du discours catalogique, différents types de temps : chronos (temps global se déployant selon une succession), aiôn (temps de vie de chacune des choses), hôra (temps conforme aux saisons), kairos (temps propice).

On peut dire, par une sorte de convention, que le «mythe » devrait rassembler, pour chaque situation, ces quatre axes. Par exemple : Ulysse est fils de Laërte (généalogie), originaire d'Ithaque (topologie), rois des Céphaloniens, engagé dans des péripéties et entouré de légendes (mythologie), se trouvant à tel moment auprès d'un tel (chronologie). C'est la façon d'articuler ensemble ces éléments, en faisant voir les souffrances et les joies, qui crée les effets affectifs, et non le fait de dire les choses successivement sur le mode d'un catalogue ${ }^{25}$. Cette conjonction n'implique pas pour autant qu'il y ait symétrie entre les axes. Dans la Théogonie d'Hésiode, les catalogues généalogiques dominent, alors que l'Iliade amplifie l'axe mythologique, même si, comme on l'a vu, elle met en œuvre des épisodes successifs régulés par le schème de la violence.

\section{Pratiques généalogiques}

De ce qui précède il ressort que la généalogie sous toutes ses formes apparaît bien comme une espèce particulière du discours catalogique mettant en jeu les trois autres axes ${ }^{26}$, et investies du schème de la parenté. Dès lors, si

\footnotetext{
${ }^{24}$ Sur ce dernier domaine, absent de notre colloque, voir S. KLIMIs, Archéologie du sujet tragique, Paris, Kimè, 2003, et S. BYL, L. Couloubaritsis (éd.), Mythe et philosophie chez Aristophane, Paris, Ousia, 1996.

${ }^{25}$ Comme le soutient, par exemple, S. Perceau au nom d'un système catalogique communicationnel..

${ }^{26}$ J'ai eu l'occasion de soutenir ailleurs que la transmutation de la civilisation archaïque vers la civilisation classique coïncide avec une promotion de la topologie au détriment de la généalogie : cosmologie présocratique au détriment de la cosmogonie, déplacements topologiques au détriment de la glorification généalogique comme élément central de la naissance de l'histoire et modifications topologiques dans l'organisation politique au détriment de la classe des eupatrides à Athènes entre Solon et Clisthène, donnant naissance à la démocratie. On pourrait ajouter qu'en philosophie, la promotion chez Platon d'une topologie paradigmatique des Idées au détriment du schème de la parenté, dégradé sur le plan épistémologique et politique, constitue un tournant dans la pensée, avec l'édification d'une science. Avec Aristote, la science a même trouvé son discours technique par la mise en forme, dans les Topiques, des lieux (topoi) du langage sur le mode de la prédication et des prédicables, avec la promotion d'un nouveau
} 
la généalogie appartient, comme l'indique à juste titre Claude Calame dans son texte introductif au colloque, aux procédés énumératifs à forte composante pragmatique qui, par la médiation de deux figures de l'accouplement et de la procréation, peut déployer progressivement une série d'entités et d'états qualifiés pour conduire à un ensemble complexe et hiérarchisé, elle est néanmoins, aussi et avant tout, un discours catalogique utilisant le schème de la parenté, associé à d'autres schèmes (violence, sexe,...). Mais dès lors qu'on touche à la genèse, la question est de savoir dans quels cas la généalogie peut être comprise littéralement et dans quels cas il faut la redresser pour rencontrer la structure du réel. Le bon sens indique que l'usage insolite d'une pratique séquentielle qui aplatit la complexité du réel en situant les termes en catalogue horizontal requiert des stratégies de décodage et d'interprétation. S’il y a démembrement du réel en faveur de pratiques séquentielles (séries, catalogues, généalogies...), il devrait y avoir redressement et reconstitution possible. En revanche, on comprend que, plus on se rapproche de l'histoire événementielle, plus l'anthropogonie autorise, jusqu'à un certain point, une lecture littérale.

Dans une analyse du mythe des races d'Hésiode, que j'ai proposée autrefois, j’ai dégagé une structure diachronico-narrative de l'ordre généalogique selon un mouvement de surdétermination, de sous-détermination et d'équilibre, en la complétant par une tentative de redressement des différentes généalogies selon un ordre hiérarchique des dieux (Théogonie), des démons, des héros, des hommes et des femmes (Les Travaux et les Jours) ${ }^{27}$. J'ai envisagé ces textes comme étant «mythiques » mais avec des ancrages dans la réalité historique, alors que d'autres considèrent, au contraire, qu'ils sont d'ordre « historique » avec des éléments mythiques. Plusieurs données légitiment ma lecture des textes.

La première provient d'un passage de la Théogonie d'Hésiode où les Muses affirment dire des mensonges (pseudea) conformes aux choses concrètes (expérimentées) et, quand elles veulent, disent la vérité (v. 27-28) ${ }^{28}$. Le procédé, fondé sur l'idée selon laquelle le «mythe » comme discours catalogique produit des distorsions, est encore pratiqué au $\mathrm{XV}^{\mathrm{e}}$ siècle. Au moment où l'hellénisme refait surface, Pléthon assigne au philosophe la mission de redresser les théogonies archaïques. Dans son Traité des Lois, il dit même qu'il faut redresser « les distorsions (diastrophas) accomplies par les poètes, au

procédé catalogique, celui des catégories, où domine la sémantique de l'agoreuô, qui signifiait déjà, chez Homère, parler en public.

${ }^{27}$ L.C. 1996 (n. 19).

${ }^{28}$ Voir aussi Odyssée XIX, 203, où le mensonge est attribué à Ulysse, qui ment à Pénélope, devenue un élément nécessaire dans sa ruse pour écarter les prétendants. En l'occurrence, Pénélope n'a pas le moyen de redresser le mensonge, comme on peut le faire pour la distorsion provoquée par le discours catalogique. Mais cela ne signifie pas qu'il n'y ait pas, en filigrane, une vérité qui puisse être redressée. C'est sur un critère de ce genre, du fait qu'il prétend que les enfants sont incapables de redresser la violence relatée dans les mythes, que Platon écarte Homère, Hésiode et les tragiques de sa Cité modèle (Rép. III). 
sens qui est le plus conforme à la philosophie »" ${ }^{29}$. L'étude de J. Fabre-Serris cidessus éclaire cette diastrophè, qui signifie non seulement « distorsion » mais aussi « perversion », et qui fait voir que la mythologie des poètes (théologiens des origines) peut-être un danger pour la religion de la cité. Cette nouvelle donnée, qui complète la première, révèle les limites d'une lecture littérale des mythes.

Une troisième donnée, issue d'un texte de Plotin, éclaire davantage le statut du discours catalogique, appliqué à l'approche généalogique. « Il faut », dit Plotin, «que les mythes, s'ils sont vraiment des mythes, fractionnent dans le temps ce qu'ils portent au discours, et divisent les uns des autres beaucoup d'êtres qui sont ensemble mais se distinguent par leur rang et leur pouvoir, là où les récits expriment par des naissances des êtres inengendrés et séparent les êtres qui sont ensemble. Mais après nous avoir instruits comme ils le peuvent, ils consentent que nous rassemblions par la pensée leurs parties successives $»^{30}$

Ce texte interprète la généalogie sur le mode du katalegein, au sens de dire successivement les choses. Aussi ai-je toujours associé, dans mes travaux, genealogein et katalegein. Mais il faut se rappeler que ce texte est du III $^{\mathrm{e}}$ siècle de notre ère et concentre des informations d'une longue histoire de la pratique du mythe. Il comprend le «mythe » comme un discours catalogique se déployant sur le mode généalogique et exprimant une réalité hiérarchique d'êtres (dont certains sont inengendrés) qui se distinguent selon deux variables au moins : rang et pouvoir. Plotin redresse ici la succession Ouranos, Cronos et Zeus selon trois hypostases hiérarchiques et éternelles : Un, Intelligence et Âme. Le terme «mythos » remplace ce qui était exprimé autrefois par «logos ». Tout en assumant une (con)fusion historique, Plotin révèle le sens d'une pratique séquentielle et catalogique, associée à la généalogie. Celle-ci traduit le démembrement du réel et sa mise en séquences afin de nous instruire sur ses configurations significatives; elle nous apprend que cette opération discursive (par le logos ou le mythos) serait elliptique si l'on ne consentait pas à opérer un redressement, "par la pensée », des parties successives, en vue de reconstituer l'ensemble.

De ce qui précède, on peut déduire une présence énigmatique et universelle des pratiques sérielles et séquentielles dans l'ordre de la parole, de l'écriture et des rites. Il est clair que ces pratiques témoignent de la nécessité d'une temporalisation selon des modes graphiques ou discursifs de succession dans le but d'éclairer la multiplicité des choses inertes et vivantes, visibles autant qu'invisibles, ou encore des événements et des situations, dont un certain nombre est d'ordre historique. Il s'agit là d'un caractère essentiel de l'activité humaine en tout temps et en tout lieu, dû aux limites objectives de la stature humaine. Par cette pratique, les êtres humains expriment et trans-

\footnotetext{
${ }^{29}$ Pléthon, Lois, p. 2 (éd. AlEXANDre, Vrin). Voir « La métaphysique de Pléthon. Ontologie, théologie et pratique du mythe », Images de Platon et lecture de ses oeuvres, Louvain / Paris, Peeters, 1997, p. 117-152; 139.

${ }^{30}$ Plotin, Enn. III, 5, 9, 24-27.
} 
crivent l'ordre du devenir en général (ce qui peut paraitre trivial) ou font voir et entendre ce qui est présent actuellement selon ses multiples dimensions en profondeur et des complexités irréductibles (ce qui est remarquable). Nous verrons que ces pratiques ne concernent pas seulement le monde ancien, mais dominent également notre civilisation, grâce aux nouvelles technologies.

Mais ce qui est le plus remarquable dans cette pratique des séries et des séquences, c'est l'instauration du discours catalogique, avec l'usage du terme katalegô, qui multiplie les possibilités discursives avec comme pratiques éminentes les généalogies et les cheminements (voyages, mais aussi les pratiques rituéliques). C'est cette dernière que je vais maintenant illustrer pour pointer la raison d'être des pratiques généalogiques (et plus spécialement de l'anthropogonie), avant de conclure par une tentative d'actualisation.

\section{Discours catalogique et schème du chemin}

Tandis que la généalogie constitue, selon ses différentes formes, un type particulier de discours catalogique, les voyages (y compris les pratiques initiatiques) intègrent le schème du chemin, comme dans le cas du retour d'Ulysse à Ithaque dans l'Odyssée homérique, de la quête de la Toison d'or dans le mythe des Argonautes ou des cycles de Thésée ${ }^{31}$. L'exemple le plus remarquable de cette extension et de son association à la généalogie se rencontre dans la séquence de la descente d'Ulysse aux enfers. Cet épisode rentre dans le cadre du séjour d'Ulysse auprès de Circé et de sa volonté de la quitter avec ses compagnons, pour retourner chez eux (Odyssée X-XI). On y découvre la souffrance et l'oubli, encadrés par les aventures d'Ulysse comme des chemins successifs dans les multiples voies qui mènent à Ithaque.

Dès l'arrivée dans l'île de Circé, Euryloque guide le premier groupe qui chemine jusqu'à sa demeure. Politès, dont l'autorité est mise en évidence, trouve la façon dont il convient de parler (muthôn) pour convaincre ses compagnons d'y entrer, en ignorant le piège tendu par Circé qui les transforme en pourceaux, au moyen d'une drogue. Flairant le piège, seul Euryloque s'échappe et rejoint Ulysse. Il raconte (katelexen) la catastrophe, c'est-àdire qu'il décrit successivement et en détail les événements (v. 224-260). Nous redécouvrons ici la distinction du katalegein et du mythos.

La suite du récit autour de la figure de Circé place Ulysse au premier plan. Avec Euryloque comme guide, il se met en route. Il rencontre Hermès qui lui assure les moyens pour détourner le plan de la déesse. Les questions que lui pose Circé s'insèrent dans le contexte archaïque : qui es-tu ? d'où viennent tes hommes? quelle est la cité d'où tu viens et celle où tu es né ? En s'étonnant du fait qu'il réussisse à neutraliser sa drogue, elle pose les conditions d'une proximité positive entre eux, qui a comme résultat la libération des compa-

\footnotetext{
${ }^{31}$ Sur Thésée, voir Cl. CALAme, Thésée et l'imaginaire athénien, Lausanne, Payot, 1990. Pour ma part, j'avais limité le schème du chemin, au début de mes recherches sur Parménide selon l'idée du savoir comme destinataire, tout en signalant l'ampleur de la question (o.c. [n. 19], p. 4142).
} 
gnons, et le séjour dans la demeure de Circé. À cette occasion surgit l'expression katalegein, mais cette fois-ci par la médiation du chœur qui lui demande de raconter successivement et en détail les événements, avant leur retour auprès de Circé (v. 421). Bien que le séjour se déroule dans les festins, la nostalgie du retour au foyer persiste. C'est à ce moment que commencent de nouvelles séquences dominées par le schème du chemin. Deux récits détaillés se suivent comme en miroir : la description des chemins par Circé (X, 488-540), puis le cheminement effectif d'Ulysse et de ses compagnons conformément à ses ordres (X, 546- IX, 50).

Circé leur indique des chemins successifs, en commençant par un premier chemin à accomplir (prôton hodon telesai) : chez Hadès et Perséphone, pour demander conseil à l'âme du devin Tirésias. À Ulysse inquiet de savoir qui les guidera sur ce chemin (bodon), Circé décrit les étapes que le vaisseau atteindra (traverser l'Océan, puis le bois de Perséphone, ensuite le chemin qui conduit à la demeure d'Hadès en passant par l'Achéron). En cet endroit, il doit accomplir un rite, en suivant des étapes précises (creuser une fosse carrée, faire trois libations avec du lait miellé, du vin doux et de l'eau pure, saupoudrer le trou d'une blanche farine, invocation des morts avec des promesses successives, offrande d'un agneau et d'une brebis noire). Résultat : les ombres des morts affluent, mais Ulysse doit les contrôler par son glaive jusqu'à l'arrivée de Tirésias qui révélera le chemin et les distances sur le chemin du retour (bodon kai metra keleuthou noston). Vient ensuite la description des voyages, qui commence par les explications d'Ulysse en ces mots : «je leur dis en utilisant cette façon de parler» (egô meta muthon eeipon, v. 561).

Or, cette description est complétée par des éléments, considérés comme interpolés, où sont décrites les ombres des figures des morts, ce qui met en scène l'ombre d'Elpénor qui déploie un plaidoyer (agoreuen), même après qu'Ulysse l'a écartée, juste avant que ne se manifestent le spectre de Tirésias, puis l'ombre de sa mère. Comme le fit auparavant Circé, Tirésias décrit à son tour les chemins à suivre et les rites à accomplir. À cette occasion le devin parle d'une série (aposteichein) complète d'hécatombes sacrées, intégrant une autre pratique séquentielle dans celle qui est dominée par le katalegein investi par le schème du chemin. Mais aussitôt Ulysse intervient et lui demande de lui dire et de lui décrire successivement et exactement en détail (tode eipe kai atrekeôs katalexon) ce qu'il en est de sa mère qui se trouve à proximité dans l'Hadès. Suit le célèbre épisode de la rencontre de sa mère Anticleia, où il lui apprend qu'il erre encore avec des souffrances, mais lui demande aussi de lui décrire une série de choses concernant les siens et ses biens, qu'il situe par des questions successives. Cette interrogation catalogique (katalegein) à laquelle répond sa mère par un discours catalogique révèle l'étendue de ce procédé, qui intègre en l'occurrence la parenté, sans qu'il s'agisse vraiment d'une généalogie.

Mais ce n'est que partie remise, du moins si l'on prend la liberté d'intégrer dans ce récit relatant la première descente aux enfers (Nekuia) la généalogie 
interpolée sous le nom des catalogues des dames (228-327) et des damnés $(328-564)^{32}$. Ulysse accueille, avec la pointe de son glaive, les ombres des reines et des princesses qu'il laisse boire successivement le sang noir, afin que chacune fasse état des exploits de sa lignée (gonon exagoreuen). Le discours catalogique qui suit associe l'axe généalogique par la mise en forme des familles de chaque figure et l'axe mythologique par les différentes formes narratives qui sont utilisées. La première partie du récit s'achève par le constat qu'il est impossible de trouver la façon d'en parler (mythèsomai), ni de les nommer toutes (passas onomènô). Suit le récit des damnés, au cours duquel Ulysse est loué par Alkinoos pour sa façon de parler (mython) qui serait à la hauteur des meilleurs aèdes, et de raconter savamment ses histoires (epistamenôs katalexas). Il l'incite à continuer de raconter exactement et successivement (atrekeôs katalexon) ses travaux et ses prodiges, car il est disposé à passer la nuit à l'écouter, à condition qu'il trouve la façon de parler de ses maux (ta sa kèdea mythèsasthai). Ulysse lui répond aussitôt : «Il y a un temps (hôrè) pour beaucoup de façons de parler (mythôn) et un temps également pour le sommeil. Si tu désires m'écouter encore je ne me refuse pas d'utiliser des façons de parler qui suscitent davantage la pitié et de faire état (agoreuein) de la mort de mes compagnons après la victoire, qui ne sont pas tombés sous le coup des Troyens..., mais au moment du retour » (v. 363384). La différence entre onomènô (je nomme un par un), katalegô (je décris ou raconte successivement et en détail), mythomai (je parle d'une certaine façon en produisant un effet) et agoreuô (je fais état publiquement) se précise. Ailleurs il est question d'arithmô (j'énumère) - ce qui donne une idée des nuances que recèle la pratique homérique du langage.

De cette expérience dans l'Hadès, il faut retenir quelque chose de plus : l'énumération des figures qui nous concernent permet d'établir aussi bien l'inventaire de celles qui s'y trouvent que leur vie dans le passé. Présent et passé se rejoignent, faisant voir aussi le sens d'une anthropogonie, liée ou non au culte des ancêtres. Elle révèle, par l'énumération et la description des traits qui s'y rapportent, le présent de leur présence actuelle dans l'invisible, et le passé de leurs exploits - dont nous pouvons faire état en les évoquant chaque fois qu'il le faut. L'important est de trouver la façon de relater ces situations, qui suscite des affections et des passions. La conjonction entre catalogues et généalogies trouve ici une raison d'être, dans la mesure où ce texte révèle que le fondement d'une généalogie est anthropogonique, et se justifie à travers l'idée que la mort n'est pas un anéantissement définitif, mais le passage dans un invisible, topologiquement déterminé (Hadès), où les spectres rassemblés peuvent être dénombrés et manifestés, sinon par des rites, du moins par un discours catalogique qui situe successivement les générations ou les morts en fonction de leur vie passée dans le visible. Dans le contexte où se tisse le paysage de l'errance et le kléos du chant, « le monde des morts

\footnotetext{
32 Comme on le sait une seconde Nekuia est décrite au chant XXIV. Cf. I. PAPADOPOULOUBelmehd, Le chant de Pénélope, Paris, Belin, 1994, p. 193-197.
} 
devient l'espace le plus proche du monde humain $»^{33}$. La pratique séquentielle sert donc ici à éclairer à la fois le présent (masse des spectres) et le passé (leur vie). Parler généalogiquement des figures du passé, c'est aussi les mettre en valeur, les porter dans la lumière en les glorifiant ${ }^{34}$. Mais, d'autre part, c'est bien par une transposition de cette pratique originaire, fondée sur le schème de proximité qu'est la parenté, que les cultures humaines ont façonné des théogonies et des cosmogonies ${ }^{35}$.

Dans cet épisode de l'Odyssée, toutes ces procédures du parler sont au moins régulées par le schème du chemin, puisque lors de son retour auprès de Circé (chant XII), après ce cheminement dans l'Hadès, celle-ci oblige d'abord Ulysse à raconter successivement les événements en départageant leur destin (kata moiran ketalexa) ${ }^{36}$, qu'elle qualifie au demeurant d'accomplissement du premier voyage. Elle décrit la suite des chemins dans les lieux appartenant aux Sirènes, où Ulysse devra choisir entre deux routes, où les obstacles se multiplient. La fin du parcours se rapproche, puisqu'au chant XIII, il atteint Ithaque.

\section{5 . Permanence des pratiques séquentielles}

Le discours catalogique régi par le schème du chemin est donc fort différent du discours catalogique régulé par le schème de la parenté qui édifie les diverses généalogies, tout comme est différent le discours mythologique de l'Iliade, dominé par le schème de la violence, discours qui est pourtant luimême déployé par une suite d'épisodes, donc par une pratique séquentielle. Aussi l'association que je m'étais permise depuis le début de mes recherches entre l'axe généalogique, l'axe mythologique, l'axe topologique et, actuellement, l'axe chronologique, même si elle me semble aller bien au-delà de toutes les tentatives proposées jusqu'ici par les exégètes, qui séparent les séries, les catalogues et les généalogies, demande à être prolongée davantage. Certes, ce qui demeure essentiel, c'est la proposition de base d'après laquelle le discours catalogique parvient à associer des choses à première vue incompatibles, comme des pratiques séquentielles qui varient en fonction des schèmes, des formes narratives sans rapport immédiat, des lieux du visible et de l'invisible, voire divers types de temps. À elle seule l'analyse séquentielle des voyages et des quêtes ouvre à des horizons insoupçonnés, une fois qu'on tient compte des pratiques initiatiques pour les rites ${ }^{37}$, des analyses actantiel-

\footnotetext{
33 PAPADOPOUlOU-BelmeHDi, ibid., p. 196

34 Il s'agit d'une question qui traverse toute mon Histoire de la philosophie ancienne et médiévale, o.c. (n. 19), p. 15-64, où je prolonge le kléos archaïque par la doxa, en retenant l'ambivalence de cette notion, qui signifie à la fois opinion et gloire.

35 Voir mes études, «Le statut transcendantal du mythe» (n. 19), p. 28 sq. et «Transfigurations du paradigme de la parenté » (n. 19), p. 173 sq.

${ }^{36}$ Cf. G. Nagy, The Best of the Acheans, Baltimore, 1979, p. 40 et 134 sq.

${ }^{37}$ Voir note 19 ci-dessus.
} 
${ }^{l e s}{ }^{38}$, des méthodes multiples, etc. Ainsi la proximité entre discours catalogique et généalogie se précise, mais en même temps confirme un divorce profond du seul fait que la pratique des catalogues atteste de multiples possibilités, tout comme la pratique des séries multiplie les applications.

Toutes ces démarches supposent au moins trois données :

1. Une distinction des différents types de procédés séquentiels : séries de choses, d'images, de graphismes, de désignations, d'actions et d'opérations (rites), diverses formes de catalogues, différentes généalogies, des cheminements variés et toutes sortes de déductions... Chacun de ces procédés présente sa spécificité et sa propre fécondité.

2. Une distinction entre une série qui exprime un devenir proprement dit (qu'il soit discursif, physique ou historique), une série qui remonte à une origine réelle ou fictive (comme le font certaines mythographies) et une série qui résulte du démembrement d'un ensemble unifié en devenir ou non (comme l'exigent la plupart des mythes). Ces trois procédés, qu'on ne peut confondre, peuvent néanmoins coexister et se recouper.

3. La possibilité, dans certain cas, et notamment dans le procédé issu d'un démembrement du réel qui est chaque fois en question, d'un redressement et d'une reconstitution de la réalité unifiée qui a été démembrée et décomposée. Mais, en général, toute pratique catalogique devrait requérir une forme de redressement, ne serait-ce que parce qu'elle implique un rassemblement conceptuel des séquences - à l'exception des déductions, transmutées en orthos logos, en discours droit, donc dressé.

Cela signifie, tout d'abord, qu'à l'instar de l'ensemble des pratiques sérielles, les catalogues et les généalogies appartiennent à une pratique séquentielle semblable, mais diffêrent par les éléments et les variables qui y sont impliqués, ainsi que par les modes de liaison qui les constituent. Cela signifie, ensuite, que toute séquence sérielle résultant d'une élucidation par démembrement d'une réalité ou d'une structure complexe, quelles qu'elles soient, doit être redressée pour reconstituer la situation initiale des choses, au risque de manquer l'essentiel, au nom d'une lecture littérale des séquences.

L'actualité des multiples méthodes séquentielles ressort de plusieurs situations : l'annuaire des téléphones, les dictionnaires et encyclopédies, les noms des morts repris sur un monument, la liste des étudiants d'une école ou d'une université, celle aussi des employés des entreprises ou des administrations, ou encore le catalogue des entreprises reprises en bourse, les inventaires du mobilier ou des produits des entreprises commerciales, le tableau périodique des éléments, la classification des minéraux et du monde vivant, les systèmes numériques en informatique, etc. Dans mon dernier livre, j'ai repris et prolongé ces perspectives dans l'ordre de la provenance des objets techniques. Cette nouvelle démarche, qui se rapproche du problème dit de la «traçabilité », traque les chaînes variables de production d'ordre technico-

\footnotetext{
${ }^{38}$ Voir à ce propos mon livre Mythe et philosophie chez Parménide (n. 19), p. 129 sq., ainsi que CALAME, o.c. (n. 31), p. 69 sq.
} 
économique, dont la description requiert nécessairement des approches séquentielles variables et hétérogènes de choses, irréductibles à la simplicité des méthodes déductives. Constitutive de la réalité visible et invisible des structures de notre époque, renforcées par l'invisible de la physique des particules et des nano-technologies, la technico-économie agit sur la vie humaine de façon qu'on puisse découvrir, parmi d'autres faits, les intérêts et les passions, les multiples souffrances provoquées par le travail, les maladies, l'exploitation, etc. C'est une conjonction analogue, mais dans un monde où l'invisible est peuplé des dieux et d'autres puissances, que nous découvrons dans les épopées homériques qui font voir, par surcroît, les joies et les souffrances des êtres humains.

Toutes ces données montrent que, pour affronter la complexité du réel, compris à la fois comme un visible et un invisible, nos cultures, à l'instar de celles du passé, choisissent encore comme démarche le démembrement et la décomposition d'une totalité de choses réunies ensemble, en relevant quelques-uns (ou le plus possible) de leurs éléments. Et pour rendre plus claire et plus efficace cette démarche, elles situent ces éléments selon des pratiques séquentielles variables. Bref, en plaçant les choses et les situations en séquences, elles réussissent à rendre visible une totalité homogène ou hétérogène impénétrable comme telle. Pour rendre encore plus spectaculaire cette question, il suffit de nous rapprocher de notre propre histoire culturelle, en nous référant à des situations insolites, auxquelles on songe rarement.

Par exemple, dans les Règles pour la direction de l'esprit, Descartes cherche à établir des lois simples grâce à des déductions à partir d'une intuition, en soulignant qu'il faut considérer les choses à connaître à partir d'un enchaînement de conséquences, d'où naissent des « séries » de choses à rechercher, auxquelles il faut ramener toute question, pour pouvoir l'examiner avec une méthode sûre $\left(\mathrm{VI}^{\mathrm{e}}\right.$ règle). Cela confirme le rapprochement que je me suis permis entre procédés séquentiels requérant un redressement et ceux qui sont déjà dressés du fait qu'ils appliquent des formes de déduction.

Plus près de nous, on peut lui opposer la pratique séquentielle analysée par Deleuze dans la Logique du sens, qui insiste sur l'importance de recouper des choses variables selon des séries, non déductives. Et entre ces deux positions, on peut situer les Éléments de théologie de Proclus, qui mettent en évidence des séries à partir des hénades divines selon un ordre d'enchaînement inspiré de la méthode dichotomique platonicienne.

Par cette actualisation aux multiples facettes, on découvre que le monde archaïque et ancien peut toujours éclairer le monde moderne. D'autant plus que nous n'avons abordé qu'un des quatre modes de l'Un et du Multiple, retenus par Aristote en Métaphysique Iota: le continu relativement aux séquences - les trois autres étant : le tout et les parties, les divers types d'universel (espèce, genre, analogie) et les choses numériquement unes ${ }^{39}$. Para-

\footnotetext{
${ }^{39}$ Voir en dernier lieu mon étude « Le statut de l'Un et du Multiple dans la Métaphysique », in M. Narcy, A. TORdesillas (éds), La Métaphysique d'Aristote, Paris, Vrin, 2005, p. 17-47.
} 
doxalement, si l'histoire de la pensée a beaucoup spéculé sur les universaux ${ }^{40}$ et sur le tout-parties ${ }^{41}$, plus récemment aussi sur la notion de singularité mais sous le prisme de l'événement (Whitehead, Deleuze, Badiou...), elle a négligé les analyses séquentielles, se limitant souvent à la notion de continu. L'esquisse que je viens de tracer montre qu'il y a encore du pain sur la planche.

Lambros COULOUBARITSIS

Université libre de Bruxelles

Centre de philosophie ancienne / CP 175/1

50, avenue Franklin Roosevelt

B -1000 BRUXeLLES

Courriel : Lambros.Couloubaritsis@ulb.ac.be

\footnotetext{
${ }^{40}$ Voir mon Histoire de la philosophie ancienne et médiévale (n. 20), p. 1030 sq., ainsi que l'étude de A. DE LiBera, La querelle des universaux. De Platon à la fin du Moyen Age, Paris, Seuil, 1996. On pourrait y ajouter les débats contemporains sur le réalisme des idées et le nominalisme des concepts.

${ }^{41}$ Souvent limité à la perspective aristotélicienne de l'étude du tout relativement aux parties, le débat a pris une nouvelle tournure depuis Husserl, et est devenu un thème majeur de la philosophie anglo-saxonne, surtout depuis l'instauration de la mériologie. Cf. mon étude " Ontologies et hénologies contemporaines », in B. Pinchard et Y. Ch. ZARKA (éds), Y a-t-il une histoire de la Métaphysique? », Paris, PUF, 2005, p. 305-343.
} 